\title{
Morphological characteristics of the deep layer of articularis genus muscle
}

\author{
E. Sakuma, Y. Sasaki, N. Yamada, I. Wada, T. Soji \\ Department of Functional Anatomy, Nagoya City University Graduate School of Medical Sciences, Nagoya, Japan
}

[Received 23 November 2013; Accepted 11 January 2014]

Background: The articularis genus muscle pulls the suprapatellar pouch upwards when the knee joint is extended, preventing mechanical impingement of the joint capsule which theoretically could cause anterior knee pain. However, few anatomical studies have addressed this muscle. Here we present the precise morphology of articularis genus.

Materials and methods: A total of 22 (13 male and 9 female) adult cadavers with no pathological conditions in the knee joints were examined during educational dissection at Nagoya City University Medical School in 2012. After exclusion of 4 joints due to their flexion contracture, 40 knee joints (18 right and 22 left) were analysed. We performed statistical analysis on anatomical laterality and the difference of sizes among lateral, medial and central branches and studied the correlation of the length and area of the articularis genus muscle to the length and cross-section area of the femur.

Results and Conclusions: The average number of branches of the deep layer of the articularis genus muscle was $2.7 \pm 0.5$, the mean length of all branches was $5.4 \pm 1.3 \mathrm{~cm}$ and the mean area of all branches was $5.5 \pm 2.6 \mathrm{~cm}^{2}$. There was no significant correlation between the length and area of the articularis genus muscle to the length and cross-section area of the femur. There was no significant laterality in central, medial and lateral branches; however we found that the medial branch was statistically longer and larger than the lateral branch on either knee. This could be contributing to prevention of lateral dislocation of the patella. (Folia Morphol 2014; 73, 3: 309-313)

Key words: muscle, anatomy, cadaver, variation, knee

\section{INTRODUCTION}

Many underlying causes have been proposed for anterior knee pain [7]. Some of these are quadriceps or patellar tendon degeneration, so-called synovial shelf disturbance and patellar chondromalacia. The anatomic structures at risk in these conditions are subchondral bone, synovium, retinaculum, skin, muscles and nerves. These structures may be affected by many factors including systemic disease but the most common reason for anterior knee pain may be inflammation brought on by overuse. One possible condition could be mechanical compression of the folds of the joint capsule including the synovial membrane within the patello-femoral joint space during joint motion $[1,5,6]$.

The capsule of the knee-joint is the largest and most extensive in the human body. The capsule is attached to the femoral bone along the edges of the shallow fossa or groove opposing the patella surface. At this point the capsule forms a deep recess known 
as the suprapatellar pouch; that forms a large cul-de-sac beneath the Quadriceps femoris on the lower part of the front of the femur. Kapandji [5] mentioned that when knee flexion is continued past $90^{\circ}$ to $135^{\circ}$ the suprapatellar pouch becomes fully unpleated. In flexion, the joint cavity becomes compressed by tension of the quadriceps femoris and the fluid moves posteriorly. When the patella moves superiorly during extension, the relatively redundant knee joint capsule would be caught between patella and femur, if not drawn upwards by the articular muscle of the knee "articularis genus muscle" [5]. This is a small muscle, usually distinct from the vastus intermedius but occasionally some branches blend with it $[1,6]$.

Kimura and Takahashi [6] reported the existence of 2 distinct layers of muscle bundles of the articularis genus muscle; the superficial and deep layer. The deep layer originates from the anterior surface of the femur and inserts into the joint capsule just proximal to the patella, while the superficial layer originates from the surface of the vastus intermedius and ends in the suprapatellar pouch. In the present study, we aim to investigate further the deep layer of articularis genus muscle [6].

The articularis genus muscle is often visualized in routine clinical magnetic resonance imaging (MRI) [8-10]. However, few anatomical studies of the articularis genus muscle have been performed and this lack of data on the normal articularis genus hampers clinical decision making in patients with related symptoms of anterior knee joint pain. To understand the function of the articularis genus muscle, its precise morphology should be studied.

This is the first report of morphometry for the deep layer of the articularis genus muscle in legs from the fixed cadavers of elderly people with emphasis on anatomical laterality and the difference of sizes among lateral, medial and central branches.

\section{MATERIALS AND METHODS}

The present study was conducted within the parameters of the written permissions we received from the donors and their surviving relatives and the present work conformed to the provisions of the Declaration of Helsinki in 1995 (as revised in Edinburgh in 2000). This study was approved by the institutional review board of Nagoya City University Graduate School of Medical Sciences according to the ethical guidelines for epidemiology research (Approved Number 810).
Table 1. Characteristics of cadavers

\begin{tabular}{|c|c|c|c|c|c|}
\hline Group & Age & Sex & $\begin{array}{l}\text { Femur length } \\
\text { [cm] }\end{array}$ & $\begin{array}{l}\text { CFS } \\
{[\mathrm{cm}]}\end{array}$ & $\begin{array}{l}\text { CSA } \\
{\left[\mathrm{cm}^{2}\right]}\end{array}$ \\
\hline 1 & 82 & $M$ & 37.5 & 9.0 & 6.4 \\
\hline 2 & 98 & $F$ & 37.0 & 9.3 & 6.9 \\
\hline 3 & 81 & $\mathrm{M}$ & 34.0 & 9.2 & 6.7 \\
\hline 4 & 88 & $\mathrm{~F}$ & 35.5 & 8.7 & 6.0 \\
\hline 5 & 73 & $\mathrm{M}$ & 37.5 & 9.0 & 6.4 \\
\hline 6 & 82 & $F$ & 36.0 & 8.0 & 5.1 \\
\hline 7 & 85 & $\mathrm{M}$ & 37.0 & 8.6 & 5.9 \\
\hline 8 & 81 & $\mathrm{~F}$ & 37.5 & 8.5 & 5.8 \\
\hline 9 & 88 & $\mathrm{M}$ & 36.5 & 8.7 & 6.0 \\
\hline 10 & 85 & $\mathrm{M}$ & 38.0 & 9.0 & 6.4 \\
\hline 11 & 95 & $\mathrm{~F}$ & 35.0 & 9.4 & 7.0 \\
\hline 12 & 81 & $\mathrm{M}$ & 35.0 & 8.8 & 6.2 \\
\hline 13 & 81 & $\mathrm{M}$ & 36.5 & 9.5 & 7.2 \\
\hline 14 & 77 & $\mathrm{~F}$ & 36.0 & 9.6 & 7.3 \\
\hline 15 & 83 & $M$ & 38.0 & 9.4 & 7.0 \\
\hline 16 & 82 & $\mathrm{M}$ & 36.5 & 9.1 & 6.6 \\
\hline 17 & 89 & $\mathrm{M}$ & 35.5 & 8.9 & 6.3 \\
\hline 18 & 84 & $\mathrm{~F}$ & 36.5 & 8.4 & 5.6 \\
\hline 19 & 89 & $\mathrm{M}$ & 39.0 & 9.2 & 6.7 \\
\hline 20 & 83 & $\mathrm{~F}$ & 35.0 & 8.2 & 5.4 \\
\hline 21 & 66 & $\mathrm{M}$ & 37.5 & 8.5 & 5.8 \\
\hline 22 & 95 & $\mathrm{~F}$ & 35.5 & 8.0 & 5.1 \\
\hline Average & 84.0 & - & 36.5 & 8.9 & 6.3 \\
\hline SD & 7.12 & - & 1.23 & 0.47 & 0.64 \\
\hline
\end{tabular}

CFS - circumference of the femoral shaft; CSA - cross-section area; F - female; $\mathrm{M}$ - male; SD — standard deviation

A total of 22 (13 male and 9 female) adult cadavers with no pathological conditions in the knee joints were examined during educational dissection at Nagoya City University Medical School in 2012. The average age at death was 81.9 years old (66-89) in male and 87.0 years old (77-98) in female (Table 1). All of the cadavers had been perfused by using fixative through the femoral artery and preserved in a natural posture in the usual manner. Their knee joints were kept in extension position where possible.

We transected and reflected inferiorly the rectus femoris and vastus intermedius to study the articularis genus muscle. After detailed dissections of the deep layer of the articularis genus muscle to make them completely visible, the morphometric measurements were taken. We recorded the location where each branch originated on the femur and designated the central branch according to its arising position in the anterior aspect of the femur, and also divided the medial and lateral branches according to their respective 
Table 2. Branch length

\begin{tabular}{|c|c|c|c|c|c|c|}
\hline \multirow[t]{2}{*}{ Group } & \multicolumn{2}{|c|}{ Medial [cm] } & \multicolumn{2}{|c|}{ Central [cm] } & \multicolumn{2}{|c|}{ Lateral $[\mathrm{cm}]$} \\
\hline & Right & Left & Right & Left & Right & Left \\
\hline 1 & 7.3 & 5.8 & 4.0 & 5.1 & 4.1 & 5.1 \\
\hline 2 & NA & 8.3 & NA & 4.8 & NA & NE \\
\hline 3 & NE & 5.9 & 6.3 & 4.2 & 4.2 & 5.0 \\
\hline 4 & 5.5 & 5.2 & 2.7 & 5.0 & 5.2 & 6.0 \\
\hline 5 & 6.1 & 6.2 & 6.9 & 4.2 & 3.5 & 4.0 \\
\hline 6 & 5.6 & 5.4 & 5.6 & 6.8 & $\mathrm{NE}$ & 3.5 \\
\hline 7 & 6.1 & NE & 4.3 & 4.2 & 5.3 & 4.0 \\
\hline 8 & 5.7 & 5.6 & 5.7 & 6.7 & $\mathrm{NE}$ & 3.2 \\
\hline 9 & NA & 8.8 & NA & 5.3 & NA & 6.3 \\
\hline 10 & 5.6 & NE & 7.8 & 5.0 & 5.6 & 5.4 \\
\hline 11 & NE & $\mathrm{NE}$ & 4.2 & 3.2 & 3.3 & 2.8 \\
\hline 12 & 5 & 7.5 & 3.3 & 4.3 & 2.9 & 4.3 \\
\hline 13 & 5 & 6 & 4.6 & 4.9 & 3.1 & 4.1 \\
\hline 14 & 5 & 5.4 & 4.3 & 4.5 & 6.0 & 3.8 \\
\hline 15 & 7.5 & 6.4 & 6.5 & 6.2 & 5.9 & 4.3 \\
\hline 16 & 7.3 & 5.5 & 7.0 & 6.0 & 4.4 & 6.5 \\
\hline 17 & NA & 5.3 & NA & 4.7 & NA & 5.8 \\
\hline 18 & NA & $\mathrm{NE}$ & NA & 6.0 & NA & $\mathrm{NE}$ \\
\hline 19 & 6.4 & $\mathrm{NE}$ & 8.4 & 7.0 & 8.4 & 5.1 \\
\hline 20 & 5.7 & 7.5 & 5.4 & 6.0 & 5.5 & 5.0 \\
\hline 21 & $\mathrm{NE}$ & 8.1 & 6.1 & 7.0 & $\mathrm{NE}$ & 4.2 \\
\hline 22 & $\mathrm{NE}$ & 5.2 & 6.6 & 6.8 & 5.5 & $\mathrm{NE}$ \\
\hline Average & 6.0 & 6.4 & 5.5 & 5.4 & 4.9 & 4.7 \\
\hline SD & 0.86 & 1.20 & 1.56 & 1.09 & 1.44 & 1.05 \\
\hline
\end{tabular}

$\mathrm{NA}$ - not available due to the knee joint flexion contracture; NE — not existing; SD - standard deviation

positions. We also recorded the distal location where it inserted into the suprapatellar pouch. Muscle length was determined as the distance between the lowest point and the highest point of each branch. However, the apparent shape and size of the articularis genus muscle is altered with the flexion position of the knee. In flexion, the suprapatellar pouch undergoes maximum stretch due to changes in the relative position of the quadriceps muscle tendon, patella, and femur. Under such circumstances, the articularis genus muscle is about twice longer than the contracted state in knee extension position $[5,6]$. Therefore, we decided that the length of articularis genus muscle should be measured in the constant position of the knee; full extension. We measured the angle of each knee joint by using an orthopaedic protractor and all joints with more than $10^{\circ}$ flexion were excluded from measurements for the length and width of articularis genus muscles. The length and width of each branch was measured by using a vernier calliper (KSM-15FF,
Table 3. Branch area

\begin{tabular}{|c|c|c|c|c|c|c|}
\hline \multirow[t]{2}{*}{ Group } & \multicolumn{2}{|c|}{ Medial $\left[\mathrm{cm}^{2}\right]$} & \multicolumn{2}{|c|}{ Central $\left[\mathrm{cm}^{2}\right]$} & \multicolumn{2}{|c|}{ Lateral [ $\left.\mathrm{cm}^{2}\right]$} \\
\hline & Right & Left & Right & Left & Right & Left \\
\hline 1 & 10.95 & 4.64 & 2.80 & 7.14 & 2.46 & 4.08 \\
\hline 2 & NA & 9.96 & NA & 4.32 & NA & NE \\
\hline 3 & NE & 5.31 & 5.04 & 4.20 & 4.62 & 6.00 \\
\hline 4 & 4.95 & 2.60 & 1.35 & 5.00 & 5.20 & 6.00 \\
\hline 5 & 6.10 & 6.82 & 11.04 & 4.62 & 3.85 & 1.60 \\
\hline 6 & 5.60 & 4.86 & 7.28 & 12.92 & NE & 3.50 \\
\hline 7 & 3.66 & $\mathrm{NE}$ & 3.01 & 5.04 & 5.30 & 2.40 \\
\hline 8 & 5.70 & 4.48 & 7.41 & 9.38 & $\mathrm{NE}$ & 2.88 \\
\hline 9 & NA & 6.16 & NA & 5.30 & NA & 3.15 \\
\hline 10 & 7.84 & $\mathrm{NE}$ & 7.80 & 5.00 & 9.52 & 8.10 \\
\hline 11 & NE & NE & 5.04 & 1.60 & 3.63 & 2.52 \\
\hline 12 & 2.00 & 7.50 & 2.64 & 3.87 & 1.16 & 4.30 \\
\hline 13 & 3.00 & 4.20 & 4.14 & 4.90 & 2.48 & 4.10 \\
\hline 14 & 2.50 & 5.94 & 5.59 & 2.70 & 4.80 & 2.66 \\
\hline 15 & 9.00 & 8.32 & 7.80 & 8.06 & 3.54 & 4.30 \\
\hline 16 & 10.22 & 4.95 & 7.00 & 6.00 & 4.40 & 6.50 \\
\hline 17 & NA & 2.65 & $N A$ & 6.11 & NA & 4.06 \\
\hline 18 & $N A$ & $\mathrm{NE}$ & $N A$ & 8.40 & NA & NE \\
\hline 19 & 8.96 & $\mathrm{NE}$ & 14.28 & 8.40 & 8.40 & 7.65 \\
\hline 20 & 2.85 & 7.50 & 6.48 & 6.00 & 2.75 & 3.50 \\
\hline 21 & NE & 8.10 & 10.37 & 7.70 & NE & 4.20 \\
\hline 22 & NE & 5.20 & 5.28 & 9.52 & 4.95 & NE \\
\hline Average & 5.95 & 5.83 & 6.35 & 6.19 & 4.47 & 4.29 \\
\hline SD & 3.01 & 2.00 & 3.26 & 2.57 & 2.18 & 1.79 \\
\hline
\end{tabular}

$\mathrm{NA}$ - not available due to the knee joint flexion contracture; NE — not existing; SD - standard deviation

Nakamura Mfg. Co., Ltd, Tokyo, Japan). We calculated the size of each branch comprising the deep layer of articularis genus muscle; length multiplied by width. Due to the flexion state of the knee joints, analysable data could not be obtained from 4 cases. After exclusion of these 4 cases, 40 knee joints (18 right and 22 left) were analysed (Tables 2, 3). We also measured the femur length and calculated the area of the cross section of the femur. The length of a femur was determined as the distance between the palpated centre of the femoral head and a point between the distal tips of the femoral condyles. We measured the circumference of the femoral shaft at the middle point by using the tape measure, and calculated the cross-section area from this circumference. Thereafter, the correlation of the length and area of the articularis genus muscle to the length and cross-section area of the femur was statistically analysed by using the Pearson product-moment correlation coefficient method. Statistical analyses on the point of laterality and the difference 
among the central, medial and lateral branches were obtained by using Student's t-test method.

\section{RESULTS}

The articularis genus muscle was present bilaterally in all of the specimens. Most muscles comprised distinct medial, central and lateral branches; however some lacked 1 or 2 of branches. The average number of branches of the deep layer of the articularis genus muscle was $2.7 \pm 0.5$, the mean length of all branches was $5.4 \pm$ $\pm 1.3 \mathrm{~cm}$ and the mean area of all branches was $5.5 \pm$ $\pm 2.6 \mathrm{~cm}^{2}$ (mean \pm standard deviation [SD]: Tables 2,3 ).

We mainly examined the deep layer of the articularis genus muscle, while we also noted the superficial layer of the articularis genus muscle during the dissection procedure.

Several lower muscle bundles of the vastus intermedius diverged from the common inserting tendon of the quadriceps femoris and inserted into the posterior surface of the suprapatellar pouch: these bundles were recognised as the superficial layer of the articularis genus muscle.

In some cases, there existed some fatty tissue which separated the deep layer from the superficial layer.

The deep layer of the articularis genus muscle ran behind the suprapatellar pouch, and the insertion of all branches was found at the level of the junction of the suprapatellar pouch and the proper joint cavity, just proximal to the patella. A fold was observed in the capsule corresponding to the insertion and traction of the muscle.

The mean length of the femurs was $36.5 \pm 1.23 \mathrm{~cm}$ and the mean of cross-section area of the femurs was $6.3 \pm 0.64 \mathrm{~cm}^{2}$ (Table 1).

There was no significant correlation between mean fascicle length and femoral length $(r=0.31)$, fascicle area and femoral length $(r=0.41)$, fascicle length and central femoral area $(r=-0.13)$, and fascicle area and central femoral area $(r=-0.09)$ by the Pearson product-moment correlation coefficient method. There was no significant laterality of fascicle length and area in central, medial and lateral branches on either knee by using Student's t-test method. However by using the same method, we found that the medial branch was statistically longer than the lateral branch on either knee (Fig. 1), and the medial branch was statistically larger than the lateral branch on either knee (Fig. 2). In addition, the central branch was statistically larger than the lateral branch on either knee $(p<0.05)$.

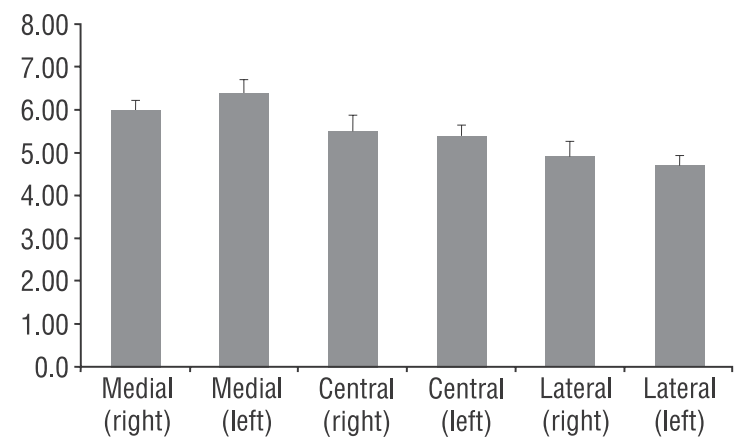

Figure 1. Branch length. Each bar represents the mean \pm standard error; medial branch: longer than lateral branch (bil: $p<0.05$ ).

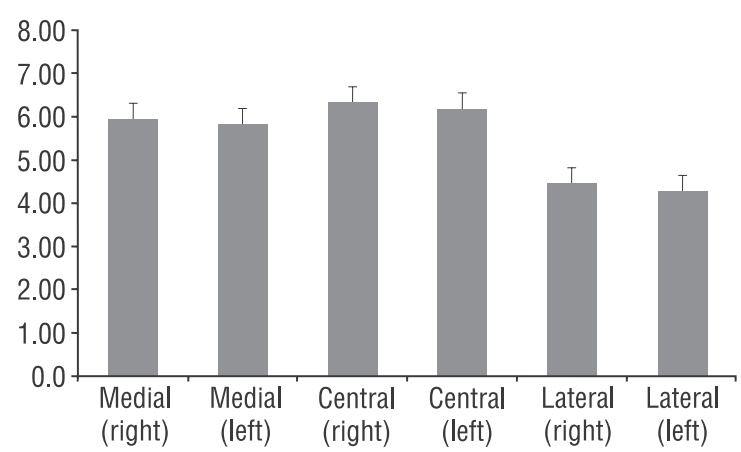

Figure 2. Branch area. Each bar represents the mean \pm standard error; medial branch: longer than lateral branch (right: $p<0.1$; left: $\mathrm{p}<0.05$ ).

\section{DISCUSSION}

The articular muscle of the knee articularis genus muscle is thought to play a crucial role in preventing the compression of the folds of the knee joint capsule. The articularis genus muscle has been thought to draw the joint capsule of the knee joint upward as the leg is extended and also known to be innervated by fibres of the femoral nerve through its branches to the vastus intermedius muscle $[1,5,6]$.

Direct evidence of this function is limited to 1 study showing that the stimulation of branches of the femoral nerve innervating the articularis genus muscle in 3 individuals who received surgical amputation caused the elevation of the capsule and suprapatellar pouch [1].

The articularis genus muscle was first described by Dupre in 1699. Thereafter, the number of muscle bundles, attachment, course and innervation of this muscle have been described by several investigators [4].

Didio et al. [2] made a study on 78 human cadavers and found the articularis genus muscle present bilaterally in all cadavers. They found that this muscle comprised 
1 or 2 bundles and inserted into the central portion of the proximal part of the suprapatellar pouch.

From a study on the knee joints of 21 cadavers and 3 living patients, Ahmad [1] concluded that the articularis genus muscle was a distinct and separate muscle from the quadriceps femoris. He reported that the muscle branch usually inserted into the central, medial or lateral aspect of the proximal border of the suprapatellar pouch, but sometimes into the anterior or posterior aspect.

Didio et al. [3] also investigated the articularis genus muscle in very young individuals focusing on morphological characteristics and microscopic appearance. He reported that it inserted into the fibrous membrane of the articular capsule of the knee. The insertion was in close association with the synovial membrane lining the proximal extension of the knee joint cavity [3].

However, the measurement technique was only briefly described in these previous reports. In a recent study by Woodley et al. [10], under a precise protocol for the detection and measurement, their dissection findings showed that the articularis genus muscle comprised a mean of 7 muscle bundles ( $7 \pm 1.8$ ). Their study showed that the muscle was approximately $14 \mathrm{~cm}$ long, which contrasts with some investigations that have estimated the muscle length to be between 6.2 and $9.0 \mathrm{~cm}$ and with our investigation in this study: the mean of number of bundles: $2.7 \pm$ \pm 0.5 and the average length: $5.4 \pm 1.3 \mathrm{~cm}$.

The disparities among the studies including the present study concerning the number of muscle branches and muscle length might reflect the methodological differences and definition rules of the articularis genus muscle. We studied only the deep layer of the articularis genus muscle, while Woodley et al. [10] investigated both superficial and deep layers.

Several reports have observed the articularis genus muscle during diagnostic imaging by MRI in volunteer subjects or in patients without anterior knee joint pain. The number and size of articularis genus muscle has also been determined in these studies. For example, Puig et al. [8] identified a mean number of $2.4 \pm$ \pm 0.7 muscle bundles and measured a mean length of $47.2 \pm 4.06 \mathrm{~mm}$ in 5 healthy young adults in a study using MRI, while Woodley et al. [10] identified $3.8 \pm 0.8$ muscle bundles in 9 cadavers by using MRI. Both of these authors commented that the articularis genus muscle could be detected in all cases, however Roth et al. [9] retrospectively studied 84 consecuti- ve patients and only identified the articularis genus muscle in $80 \%$ (74/92 knee joints).

In the present study, we tried several statistical analyses and found that the medial branch was statistically longer and larger than the lateral branch on either knee (Figs. 1, 2). Normally the patella moves mainly in the sagittal plane and not transversely. It is in fact very strongly applied to its groove by the quadriceps, the more so as the degree of flexion increases. At the end of extension this appositional force is diminished and in hyperextension it even tends to be reversed to separate the patella from the femur. At this point the patella tends to be driven laterally because the quadriceps tendon and the ligamentum patellae form an angle obtuse laterally. Lateral dislocation of the patella is mainly prevented by the lateral lip of the patellar surface of the femur which is distinctly more prominent than the medial lip [5].

\section{CONCLUSIONS}

In the present study, the statistically longer and larger medial branch of the deep layer of articularis genus muscle could be contributing to prevention of lateral dislocation of the patella.

\section{REFERENCES}

1. Ahmad I (1975) Articular muscle of the knee: articularis genus. Bull Hosp Joint Dis, 36: 58-60.

2. Didio U, Zappala A, Carney WP (1967) Anatomico-functional aspects of the musculus articularis genus in man. Acta Anat (Basel), 67: 1-23.

3. Didio U, Zappala A, Cardoso AD, Diaz RA (1969) Musculus articularis genus in human fetuses, newborn and young individuals. Anat Anz, 124: 121-132.

4. Hyrtl J (1859) Dupre: Les sources de la synovie (1699); Lehrbuch der Anatomie des Menschen mit Rücksicht auf physiologische Begründung und praktische Anwendung. 6 Ed. Braumüller, Paris, p. 438.

5. Kapandji IA (1970) The physiology of the joints. Vol 2. Lower limb. Honore LH tr. Churchill and Livingstone, Edinburgh, pp. 92-103.

6. Kimura K, Takahashi YM (1987) articularis genus. Observation on arrangement and consideration of function. Surg Radiol Anat, 9: 231-239.

7. Post WR (2005) Anterior Knee Pain: diagnosis and treatment. J Am Acad Orthop Surg, 13: 534-543.

8. Puing S, Dupuy DE, Sarmiento A, Boland GW, Grigoris $P$, Greene R (1996) Articular muscle of the knee: a muscle seldom recognized on MR imaging. Am J Roentgenol, 166: 1057-1060.

9. Roth C, Jacobson J, Jamadar D, Caoili E, Morag Y, Housner J (2004) Quadriceps fat pad signal intensity and enlargement on MRI: prevalence and associated findings. Am J Roentgenol, 182: 1383-1387.

10. Woodley SJ, Latimer CP, Meikle GR, Stringer MD (2012) Articularris genus: an anatomic and MRI study in cadavers. J Bone Joint Surg Am, 94: 59-67. 